\title{
Swift and Energy Efficient Big Data Gathering Approaches in Wireless Sensor Networks
}

\author{
T. Sujithra \\ Research scholar \\ Department of Computer Science and Engineering \\ PSG College of Technology, Coimbatore, India
}

\author{
R. Venkatesan \\ Professor \\ Department of Computer Science and Engineering \\ PSG College of Technology, Coimbatore, India
}

\begin{abstract}
In Recent studies, mobile element acts as a mechanical carrier equipped with a powerful transceiver and battery. It directly collects the data from the sensors in the sensing environment via single-hop communication when traversing its transmission range and eventually delivers the collected data to the remote central. As a mobile element collects the data from every sensor node, the length of the mobile element tour will be increased. It results in increased data gathering latency. To solve this problem, several algorithms have been proposed. One of them called Toward Energy Efficient Big Data Gathering (TEEBD). Even it simplifies the mobile element data gathering by calculating the optimum number of clusters. Mobile element should wait until all of its cluster members uploads its data. It gives increased data gathering latency, and Packet loss due to buffer flow. In this paper, we propose two novel approaches called Energy Efficient Big Data Gathering using Local data Collector (EEBDG-LC) and Energy Efficient Big Data Gathering using Local data Collector with Threshold (EEBDG-LCWT). First approach concentrates on placing a local data collector in every centroid of the region. In which mobile element collects the information only from local data collector instead of all of its sensor nodes. It increases the speed of mobile element data gathering. The main goal of the second approach is to reduce the traffic in the local sensing region of EEBDG-LC based on the threshold value. In which node reaches the threshold value are only allowed to transmit data to the local data collector. Others go to the sleep mode immediately. Thus, increases the lifetime of the sensor network, and packet delivery ratio. Various data gathering mechanisms such as mobile element data gathering and data gathering using UAV have been used and comparison between these two has been done. The effectiveness of our approach is validated through extensive simulations.
\end{abstract}

\section{Keywords}

Local data collector, Unmanned Arial Vehicle, Threshold, Mobile Element.

\section{INTRODUCTION}

Wireless sensor networks (WSNs) are emerging as a radical technology. Integration of simple processing, memory, sensing and communication capabilities into small scale low cost devices and connecting them into so-called wireless sensor networks opens the doorway to a glut of new applications [1]. The technology for sensing and control has the potential for significant advances, not only in science and technology, but equally important for a broad scope of applications pertaining to wellness maintenance, energy, critical infrastructure protection and security, environment, food safety, production processing, quality of life and economy [2]. Energy efficiency plays a pivotal role in wireless sensor network as it affects the lifetime of the sensor networks. Furthermore, once the sensor nodes are deployed, it is hard to recharge due to either the huge volume of sensor node deployment or human non-intervention characteristics of the deployment area. In a wireless sensor network, most of the energy is consumed for communication purpose. The amount of energy consumed in wireless communication is directly proportional to the communication distance. Therefore, the long distance communication is not preferred. Usually, the data collection in large geographical area is accomplished via multi-hop communication. Furthermore, the expenditure of energy in multi-hop communication is heterogeneous as nodes near the base station (BS) drain out very quickly. Because, these nodes are forced to forward the data of other nodes in the network to BS. In order to address the non-uniform consumption problem among the sensor nodes, recently researchers have been introduced mobile element (ME) [3]. ME could be a mechanical agent equipped with a powerful transceiver and battery. It directly collects the data from the sensors in the sensing environment via single-hop communication when traversing its transmission range and finally delivers the collected data to the data collection centre. As a ME collects the data from every sensor node, the length of the ME tour will be increased. However, the lifetime of the network increases by avoiding multi-hop communication. Data gathering latency is high. Hence this approach is not suitable for time sensitive applications. In order to reduce the data gathering latency, some of the researchers introduced local data collection points called rendezvous points (RPs) [4]. These rendezvous points temporarily cache the sensing data and deliver the data to ME when it arrives. Although these approaches effectively reduce the latency of ME data gathering, the lifetime of the sensor network is not taken into account. In this paper, we propose a novel way of data gathering mechanism for time sensitive applications using single ME. The main contribution of this paper is to

- Remove the overhead of intra region information exchanges.

- Remove the time synchronization between ME and sensors.

- Causes reduced data gathering latency and improves the network lifetime compared to the benchmarking system.

The rest of the paper is organized as follows. Section 2 describes the related work. Section 3 discusses about the proposed scheme. Section 4 deals with the experimental results and analysis. Finally, section 5 concludes this paper.

\section{RELATED WORK}

This section discusses about the works that are under study leading to fresh estimates. Based on the sensor node deployment, wireless sensor network is classified into two, 
namely homogeneous network and heterogeneous network. Sensor nodes deployed in a homogeneous network having the identical communication capability and equal amount of energy, in which some of the nodes are selected as cluster head, because of energy insufficiency role of cluster head is rotated, however energy consumption is eventually distributed among the sensor nodes. Frequent cluster head re-election gives additional overhead [5] [6]. Heterogeneous network composes of small number of resource rich nodes and large number of energy limited basic sensor nodes. The basic sensor node has only limited communication capability and energy. Resource rich nodes are equipped with powerful transceivers and high battery. In which resource rich nodes are acting as cluster head and basic sensor nodes are acting as cluster members. However, cluster head re-election frequency is minimized by excessive energy. Placing resource rich nodes at appropriate location is difficult [7]. Event driven, query based, time driven and hybrid are possible ways of initiating the data transfer from sensors to the sink [8]. For any data gathering scheme, sensors should send their readings to the sink, it may be either static sink or mobile sink. Mostly in static sink approach, data are transferred from sensors to the sink via multi-hop communication. Due to this, nodes near the static sink deplete its energy quickly. It makes the static sink unreachable. In mobile sink approach, mobile sink roams the sensing environment and visits every sensor node physically in its transmission range and collects the data from it via single-hop communication. Sink mobility decides the communication pattern between the sensor nodes and the sink. Based on the trajectory of the mobile sink, sink mobility can be classified into three types such as random path, constrained path and controlled path [9]. In random path approach, mobile sink uses the random path for visiting the sensor nodes. In this approach, mobile sink is fixed on the body of an animal and moving randomly to collect the required information sensed by the sensor nodes. Because of random movement of an animal, mobility is hard to predict. Thus, the data gathering latency cannot be guaranteed. In Constrained path approach, mobile sink moves along a fixed path to collect the information from the sensor nodes. In Controlled path approach, mobile sink visits the sensor nodes by using the computed optimal path. Visiting all the sensor nodes in the network by using single mobile sink increase the data gathering latency. In order to overcome this, rendezvous points are introduced. Rendezvous point is a point responsible for gathering the data from the source nodes and buffering the aggregated data until ME visits. Three heuristic algorithms have been discussed in [10]. The first algorithm, earliest deadline first (EDF) algorithm implies that the node with the closest deadline is visited first. EDF with k-lookahead considers the $\mathrm{k}$ ! permutations of the $\mathrm{k}$ nodes with smallest deadlines, and chooses the next node which leads to the earliest finish time consequently. Third algorithm, minimum weight sum first (MWSF) considers both deadlines and distance between nodes in finding the visiting schedule. Threshold value plays a key role in controlling data transmission. Data is transferred only when sensing value reaches the threshold, otherwise it would not send any data to BS. It reduces the amount of data transmission [11]. The work in [12] discusses about the energy efficient big data gathering using single mobile element. In which, the entire sensing area is partitioned into equal sized regions. Nodes within the region are grouped together based on the degree of dependence called responsibility. K-Means algorithm is used to find the centroid of the cluster. Data is gathered at the centroid of the cluster. ME visits every centroid of the cluster and gathers the data from all of its nodes in the cluster.
Travelling salesman problem (TSP) algorithm is used to find the optimum travelling path of the mobile element. Say Sotheara et al. proposed effective data gathering and energy efficient communication protocol in wireless sensor networks employing UAV [13]. It concentrates on reducing the number of nodes that are communicating with the UAV. It divides the coverage area of the beacon signal into frames with different priorities. Frames are classified as frames that are in the farthest back side and frames that are in the farthest front side. Frames that are in the front side getting highest priority than the backside frames. Data is gathered from highest priority frame to lowest priority frames. In [14] forest fire modelling and early detection system was presented. The main idea is to design a system for early forest fire detection using fire weather index (FWI) system. FWI is one of the fire rating systems used in North America. Temperature and humidity are considered in analyzing the fire index behaviour. Indexes of FWI system are classified as initial spread index (ISI), build up index (BUI) and fire weather index. FWI is calculated based on ISI and BUI. It mainly concentrates on two major components such as a fine fuel moisture code (FFMC) and FWI. FFMC provides the early warning of potential fire. FWI gives magnitude and intensity of the forest fire. Range of FFMC and corresponding fire intensity as per their results is presented as below

Table 1. Intensity Level of Fire based on FFMC

\begin{tabular}{|c|c|}
\hline Range of FFMC & Fire Intensity \\
\hline $0-76$ & Low \\
\hline $77-84$ & Moderate \\
\hline $85-88$ & High \\
\hline $89-91$ & Very High \\
\hline $92+$ & Extreme \\
\hline
\end{tabular}

In this work, we focus on energy efficient big data gathering approaches for time sensitive application. Our work is based on the work [12], in which a single ME is responsible for entire network data gathering. Due to this, data gathering latency is increased. Hence it is not suitable for time sensitive application. The delayed data gathering also gives loss of data because of its buffer size. Tight synchronization is needed between ME and the sensor nodes in the sensing environment. Cluster formation gives additional overhead. The main goal of our paper, is to increase the speed of data gathering by introducing local data collector in every region and increase the packet delivery ratio by controlling the communication between the local data collector and the sensor nodes in the sensing environment based on the threshold value. Finally, improves the network lifetime by efficient transceiver scheduling.

\section{PROPOSED PROTOCOLS}

\subsection{Energy Efficient Big Data Gathering using Local data Collector (EEBDG- LC)}

The proposed energy efficient big data gathering using local data collector approach is designed based on the following observations. Assume that a WSN has been designed for forest fire detection application. WSN has three mandatory

components: basic sensor nodes, special sensor nodes and ME. Some of their characteristics in WSN are listed below. 
- Sensing environment composes of a large number of basic sensor node and limited number of special sensor nodes.

- The basic sensor nodes are stationary. It has only limited energy and communication capabilities.

- The special nodes are mobile. It has a powerful transceiver. It is equipped with energy harvesting source.

- Basic sensor nodes can not be recharged after deployment.

- ME is the base station fixed on a moving object.

- ME moves with a fixed velocity on a computed path.

- TSP algorithm finds a path for ME visit.

- Location of the local data collector is known.

- Lowest value of FFMC in Table 1. is set as threshold value $\left(\mathrm{t}_{\mathrm{h}}\right)$.

- Toward energy efficient big data gathering approach [12] is taken as a benchmarking system.

\subsubsection{Problem identification}

In [12], a single mobile element is used to collect the information from large scale environment. The main idea of this approach is to find the optimum number of clusters for large scale data gathering. Mobile element data gathering is carried out at the centroid of the cluster instead of visiting the cluster members in its transmission range. Even it finds optimum clusters for data gathering, ME should wait until all of its cluster members uploads its data. It gives delayed data gathering, packet loss because of buffer overflow, and affects the lifetime of the network because of multi-hop communication. Now, as an extension to this work, we propose a novel approach named Energy Efficient Big data gathering using local data collector. At the outset, the entire sensing area is partitioned into equal sized regions. The main idea of this approach is to introduce the special sensor node called local data collector in every centroid of the region. In which ME collects the information from only the subset of nodes called local data collector instead of collecting information from all of its sensor nodes. It improves the speed of ME data gathering.

\subsubsection{Algorithmic solution}

Our first proposed approach EEBDG-LC concentrates on region partitioning, placement of local data collector, and $\mathrm{ME}$ data gathering.

\subsubsection{Region partitioning}

Multi-hop communication is one of the best ways to improve the network lifetime. Even it improves the network lifetime, nodes near the sink run out of energy very soon. Hence, the distance between the data sources and the sinks are very important while designing WSN. The energy level of the data collector is very important, because poor data collector selection results frequent re-election, it gives additional overhead. Based on the above points, we design our WSN. We assume, sensing area (A) composes of the limited amount of special sensor nodes and a large amount of basic sensor nodes. Let $\alpha$ be the communication range of the special sensor node (local data collector) and $\beta$ be the communication range of the basic sensor node where $\alpha>\beta$. In order to reduce the multi-hop communication between the data sources and local data collector, the entire sensing area (A) is partitioned into equal sized smaller regions $(\mathrm{R})$. The size of the region depends on the communication range of the special sensor node. It is denoted as
$A \rightarrow R$

$R=\left\{r_{1}, r_{2}, r_{3}, \ldots ., r_{n}\right\}$ and $\left|r_{1}\right|=\left|r_{2}\right|=\left|r_{3}\right|=\ldots=\mid r(1)$

$A=m(2 \alpha)$ Where ' $\mathrm{m}$ ' is constant

\subsubsection{Placement of local data collector}

Local data collector (LDC) is a special sensor node equipped with energy harvesting source. In order to reach all of its sensor nodes in its local sensing region effectively, placement of local data collector is very important. The local data collector is positioned based on K-Means algorithm. It is one of the conventional algorithms to find the centre position of the region. As in K-Means algorithm, initially it is placed randomly in any one of the place in the local sensing area as follows

$L=\left\{l_{1}, l_{2}, l_{3}, \ldots \ldots, l_{n}\right\}$

$K=\left\{k_{1}, k_{2}, k_{3}, \ldots \ldots, k_{s}\right\}$

Where $1_{1}, 1_{2}, 1_{3}, \ldots \ldots ., 1_{n}$ denote local data collectors available in total sensing area, and $\mathrm{k}_{1}, \mathrm{k}_{2}, \mathrm{k}_{3}, \ldots \ldots \ldots \mathrm{k}_{\mathrm{s}}$ denote local sensing area. Initial location of the local data collector $l_{i}$ is denoted as

$k_{i}\left(l_{i}\right)=\left(x_{j}, y_{j}\right), k_{i} \in K, l_{i} \in L$ and $\left(x_{j}, y_{j}\right) \in m X n$

where $(\mathrm{mXn})<(\mathrm{NXN}) . \mathrm{mXn}$ denotes the boundary of local sensing area $\left(\mathrm{k}_{\mathrm{i}}\right)$ and NXN denotes boundary of the total sensing area (A). After that, the local data collector is repositioned to the centre position of the local sensing region $\left(\mathrm{k}_{\mathrm{i}}\right)$ by applying K-Means algorithm recursively until it is placed optimally.

$\left.L_{n}=\left\{j: d\left(x_{j}, \mu_{i}\right) \leq d\left(x_{j}, \mu_{1}\right), 1 \neq i, j=1, \ldots, n\right)\right\}$

Where $L_{n}$ denotes the point which is closest to the center position of the local sensing area.

$\mu_{i}=1 /\left|n_{i}\right| \Sigma j \in n_{i} x_{j}, \forall I$

where $\mu_{\mathrm{i}}$ is the mean value of the particular region and $n_{i}$ is total number of nodes in the region which produces new location

$k_{i}^{\prime}\left(l_{i}\right)=\left(x_{i}^{\prime}, y_{i}^{\prime}\right)$

\subsubsection{Data gathering}

TSP algorithm to find ME tour for given set of local data collectors. Once ME tour is constructed, ME starts collecting the information from the local data collector over the path computed by TSP.

\subsection{Energy Efficient Big Data Gathering using Local Data Collector With Threshold (EEBDG-LCWT)}

It is an enhanced version of EEBDG-LC. It integrates the features of EEBDG-LC with the threshold. The main objective of this approach is to reduce the data traffic in the local sensing region and improve the network lifetime by controlling the communication between the local data collector and the sensor nodes in the local sensing region based on the sensor node classification. The sensor nodes in the local sensing region are classified based on the threshold value as imminent nodes and silent nodes. The nodes reaches the threshold value called as imminent nodes that are only allowed to remain in the transmit mode and delivers the data to the local data collector, others goes to the sleep mode immediately. Thus transceiver scheduling improves the network lifetime. A value that needs to measure from the environment is set as the threshold. Threshold value varies from one application to another. It is application dependent. For implementation purposes, we take the reference of forest fire detection application to set as threshold value. The lowest value of FFMC in Table.1 is set as the threshold. This value broadcasts to all the nodes in the sensing environment at the 
time of region formation by ME. Whenever the requirement changes, the threshold value is refreshed. It reduces the amount of data which is to be transferred from the sensor nodes to the local data collectors which results in the reduction of data traffic inside the local sensing area.

\subsubsection{Classification of sensor nodes in the local sensing area}

Initially, all the nodes in the local sensing area $\mathrm{k}_{\mathrm{i}}$ are kept in idle mode that is represented as

$N_{i}=\left\{n_{1}, n_{2}, n_{3}, \ldots \ldots, n_{p}\right\}$

Where $\mathrm{N}_{\mathrm{i}}$ denotes total number of nodes in local sensing area $\mathrm{k}_{\mathrm{i}}$.

$\forall n_{i} \in N_{i}: n_{i} \rightarrow$ idle

Nodes whose sensing value that reaches the threshold value $\left(t_{h}\right)$ is switched over into the transmit mode and others goes to the sleep mode immediately. It is represented as

$S_{i}=\left\{s_{1}, s_{2}, s_{3}, \ldots . . ., s_{t}\right\}$

Where $S_{i}$ denotes the sensing value of the sensor nodes $\left(n_{i}\right)$ in the local sensing area $\left(\mathrm{k}_{\mathrm{i}}\right)$

$\exists s_{i} \in t_{h}: n_{i} \rightarrow t_{\text {mode }}$

where $t_{\text {mode }}$ denotes the transmit mode. Data is collected only from the imminent nodes. It reduces the data traffic in the local sensing region and automatically increases the lifetime of the sensor nodes.

\subsubsection{Algorithm}

Input : Result of EEBDG-LC.

Output : Reduced data traffic and increased network lifetime.

Goal : To reduce the data traffic inside the local sensing area $\mathrm{k}_{\mathrm{i}}$ using Threshold.

1. Feed local sensing area $\mathrm{k}_{\mathrm{i}}$ with properly placed local data collector $l_{i}$ and randomly deployed sensor nodes to EEBDG-LCWT.

2. Initialize the threshold value $t_{h}$.

3. Broadcast the threshold value to all sensor nodes in the sensing region.

4. Schedule the states of the transceiver for $n_{i} \in N_{i}$

$$
\begin{aligned}
& \text { if } \mathrm{s}_{\mathrm{i}}>\mathrm{t}_{\mathrm{h}} \\
& \quad \mathrm{n}_{\mathrm{i}}=\mathrm{t}_{\text {mode }} \\
& \text { else } \\
& \mathrm{n}_{\mathrm{i}}=\text { sleep }
\end{aligned}
$$

5. LDC collects the data from only the imminent nodes.

\subsection{Swift and Energy Efficient Big Data Gathering (SEEBDG)}

Various data gathering approaches are available for collecting the information from the sensing environment like mobile element data gathering, data gathering using UAV, multi-hop data gathering etc., It is an enhanced version of the EEBDGLCWT. In order to improve the fastness of the data gathering further, we propose swift and energy efficient big data gathering approach. In which unmanned arial vehicle is used for data gathering instead of ME. . It reduces the delay in the data gathering process compared to the mobile element data gathering approach. For gathering the data, UAV broadcast the beacon signal periodically. Once the local data collector receives the beacon signal, it starts to transmit the data to the UAV.

\subsubsection{Movement of $U A V$}

The traversal path of the UAV depends on the coverage area of the beacon signal. Initially, the coverage area of the beacon signal is divided into many different frames with different transmission priority. Frames categorized into two, namely frames that are in farthest front side and the frames that are in the farthest back side as in [13]. Frames that are in the farthest front side are getting higher priority than frames that are in the furthest back side. Data is gathered from the highest priority frame to the lowest priority frames. UAV collects the data from the local data collector who receives the beacon signal of UAV. It results faster communication compared to mobile sink approach.

\section{EXPERIMENTAL RESULTS AND ANALYSIS}

This section presents the evaluation of swift and energy efficient big data gathering approaches with respect to the following performance metrics; (1) The lifetime of the network (2) Delay in data gathering and (3) Packet delivery ratio. We look the parameters considered in our experiments by varying the number of nodes as $20,30,40,50,60,70,80$, 90,100 . Table 2 . summarizes important simulation parameters and their default values as shown in below.

Table 2. Simulation Settings

\begin{tabular}{|c|c|}
\hline No. of nodes & $20,30,40,50,60,70,80,90,100$ \\
\hline Node deployement & Random \\
\hline Mac & 802.11 \\
\hline Total sensing area & $500 \times 500 \mathrm{~m}$ \\
\hline Communication Range of $\beta$ & $30 \mathrm{~m}$ \\
\hline Communication Range of $\alpha$ & $60 \mathrm{~m}$ \\
\hline Initial Energy & $100 \mathrm{~J}$ \\
\hline Transmit Energy & $0.02 \mathrm{~J}$ \\
\hline Receive Energy & $0.01 \mathrm{~J}$ \\
\hline Threshold (th) & 76 \\
\hline
\end{tabular}

Below we discuss each performance metric in detail.

1) Data gathering latency : It is the time taken by ME to collect the information from the data sources.

2) Packet delivery ratio : It is defined as the number of data packets received successfully with the total number of packets sent.

3) Energy consumption : It is the total energy consumed by the nodes in receiving and sending the packets.

Fig. 1.a shows the performance of four algorithms as a function of density of sensor nodes, in terms of energy consumption. For, TEEBD, EEBDG-LC, and EEBDGLCWT, ME is used for data gathering. For, SEEBDG, the UAV is used for data gathering. In Fig 1.a, it is easy to observe that EEBDG-LC outperforms than the TEEBD. The reason behind this, In TEEBD, ME collects the information from all of its cluster members. It makes all the sensor nodes to be in active mode. Furthermore, information is transmitted to $\mathrm{ME}$ via multi-hop communication. It deteriorates the lifetime of the sensor network. In EEBDG-LC, region partitioning is done based on the coverage area of the LDC. 
Furthermore, it is placed at the center of the region. Hence, multi-hop communication is reduced. It increases the network lifetime. Energy consumption of EEBDG-LCWT is minimal compared to EEBDG-LC. The reason for this is, the imminent nodes are only allowed to remain in the transmit mode and deliver the data to LDC. Others, goes to the sleep mode immediately. Thus transceiver scheduling improves the network lifetime. EEBDG-LCWT and SEEBDG gradually decreases the energy consumption and then stabilizes when the density of the nodes becomes large.

Fig. 1.b plots the performance of four algorithms as a function of density of sensor nodes, in terms of data gathering latency. It shows that, EEBDG-LC greatly reduce the data gathering latency than TEEBD. The reason is, in TEEBD, ME collects the information from all of its cluster members. It increases the data gathering latency. In EEBDG-LC, ME collects the information from only the local data collectors instead of all of its sensor nodes in the sensing environment. Hence, the data gathering latency is reduced. Furthermore, there is no need for tight synchronization between the sensor nodes and ME. Data gathering latency is further improved in SEEBDG. Because, it uses UAV to collect the information. Fig. 1.c plots the performance of four algorithms as a function of density of sensor nodes, in terms of packet delivery ratio. It shows that, packet delivery ratio is improved in EEBDG-LC than TEEBD. The reason is, in TEEBD, ME collects the information from all of its cluster members which increase the data traffic inside the cluster. It results in packet loss. In EEBDG-LC, LDC collects the information from the sensor nodes via single-hop communication because of optimum placement of local data collector. The packet delivery ratio is further improved in EEBDG-LCWT. The reason is, communication between the LDC and sensor nodes are controlled based on the threshold value. LDC collects the information only from the imminent nodes instead of all. It reduces the data traffic in the local sensing region and improves the packet delivery ratio. It is further improved in SEEBDG, because it increases the speed of data gathering by using the UAV. It controls the packet loss because of buffer overflow.

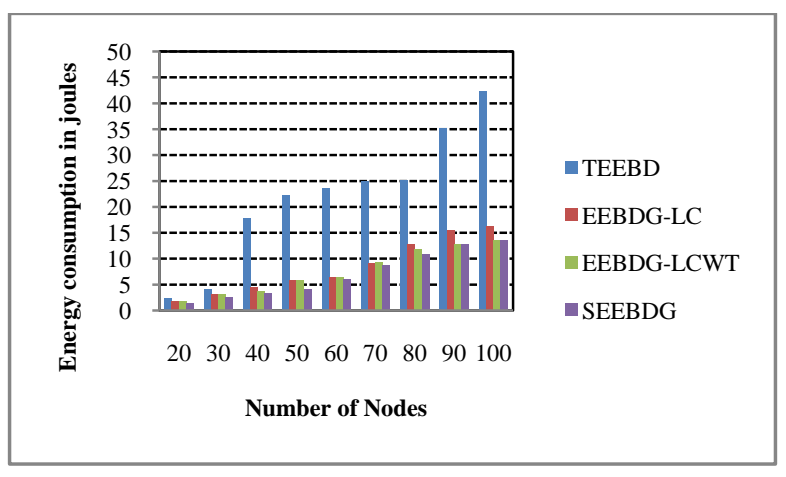

Figure. 1.a Energy Consumption Vs Number of Nodes

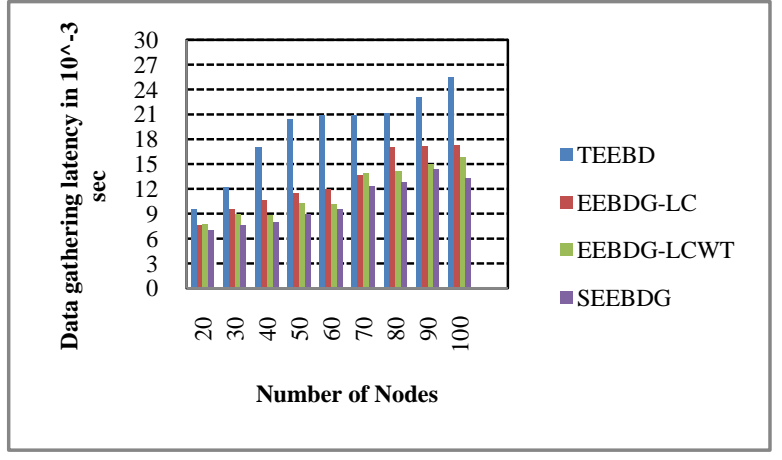

Figure. 1.b Data gathering latency Vs Number of Nodes

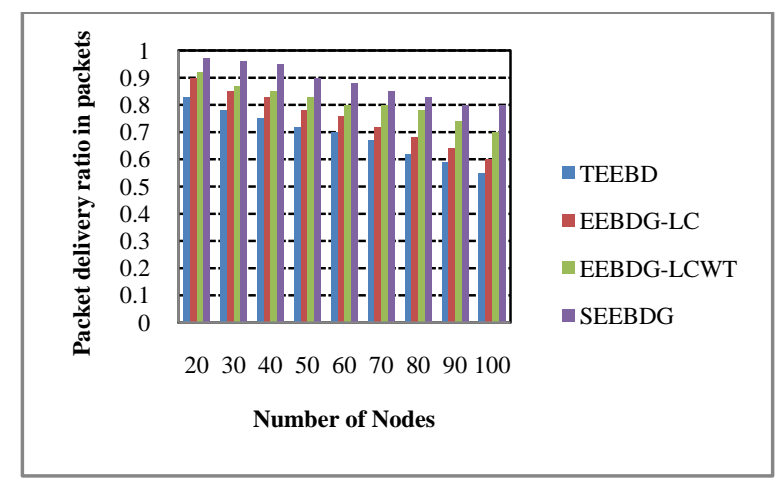

Fig. 1.c Packet delivery ratio Vs Number of Nodes

\section{CONCLUSION}

In this paper, we have considered different issues such as data gathering latency, energy consumption and packet delivery ratio occurred during the massive data gathering. For reducing packet loss, data gathering latency and improving the lifetime of the sensor network, we have proposed two protocols namely EEBDG-LC, EEBDG-LCWT. In EEBDG-LC, sensing area is partitioned into smaller regions based on the coverage area of the LDC. It reduces the multi-hop communication. A local data collector is placed in every local sensing area based on $\mathrm{K}$-Means algorithm for gathering the data from its local sensing region. Because of its energy independence, re-election overhead is avoided.In which, ME collects information only from the local data collector instead of all of its sensor nodes. It improves the speed of ME data gathering. This is further improved by incorporating the feature of EEBDG-LC with threshold called EEBDGLCWT.It controls the communication between the local data collector and the sensor node based on the node classification. LDC collects the information only from the imminent nodes instead of all of its sensor nodes. It reduces the data traffic in the local sensing region and improves the network lifetime by efficient transceiver scheduling. We use various data gathering approaches to collect information and extensive simulation is carried out. From the simulation, we have shown our proposed protocol outperforms than the benchmarking system in terms of energy consumption, packet delivery ratio and data gathering latency.

\section{REFERENCES}

[1] Holger Karl and Andreas Willig, "Protocols and architectures for wireless sensor networks," John Wiley, England, 2005.

[2] Kazem sohraby, Daniel Minoli and Taieb Znati, "Wireless Sensor Networks: Technology, Protocols, and 
Applications," Wiley Interscience, United States of America, 2007.

[3] J. Luo, J. Panchard, M. Pi'orkowski, M. Grossglauser, and J.P. Hubaux, "MobiRoute: routing towards a mobile sink for improving lifetime in sensor networks," In . 2nd IEEE/ACM DCOSS, pp. 480-497, Springer, 2006.

[4] Guoliang Xing, Tian Wang, Zhihui Xie, and Weijia Jia, "Rendezvous Planning in Wireless Sensor Networks with Mobile Elements," In. IEEE transactions on mobile computing, pp. 1430-1443, 2008.

[5] W. R. Heinzelman, A. Chandrakasan, and H. Balakrishnan, "Energy efficient communication protocols for wireless micro sensor networks," In . Proc. HICSS, pp. 1-10, 2000.

[6] X. Liu, J. Cao, S. Lai, C. Yang, H. Wu, and Y. Xu, "Energy efficient clustering for WSN based structural health monitoring," In. Proc. IEEE INFOCOM, pp. 2768-2776, 2011.

[7] Z. Zhang, M. Ma, and Y. Yang, "Energy efficient multihop polling in clusters of two-layered heterogeneous sensor networks," In. IEEE Transtions on Computer, pp. 231-245, 2008

[8] T. A. A. Alsbou, M. Hammoudeh, Z. Bandar, and A. Nisbet, "An overview and classification of approaches to information extraction in wireless sensor networks," In. Proceedings of the 5th International Conference on Sensor Technologies and Applications (SENSORCOMM '11), pp. 255, 2011.

[9] M. Di Francesco, S. K. Das, and G. Anastasi, "Data collection in wireless sensor networks with mobile elements: a survey," In. ACM Transactions on Sensor Networks, pp. 7:1-7:31, 2011.
[10] A.A. Somasundara, A. Ramamoorthy, M.B. Srivastava, "Mobile element scheduling for efficient data collection in wireless sensor networks with dynamic deadlines," In. proceedings of the 25th IEEE International Real-Time Systems Symposium (RTSS'04), pp. 296-305, 2004.

[11] Manjeshwar and D. Agrawal, "Teen: A Routing Protocol for Enhanced Efficiency in Wireless Sensor Networks," In. IEEE parallel and distributed processing symposium (IPDPS), pp.2009-15, 2001.

[12] Daisuke Takaishi, Hiroki Nishiyama, Nei Kato and Ryu Miura, "Toward Energy Efficient Big Data Gathering in Densely Distributed Sensor Networks," In. IEEE Trans. Emerging Topics in Computing, pp. 388-397, 2014.

[13] Say Sotheara, Kento Aso, Naoto Aomi, and Shigeru Shimamoto, "Effective Data Gathering and Energy Efficient Communication Protocol in Wireless Sensor Networks employing UAV', In. IEEE WCNC'14, Track $3,2014$.

[14] Mohamed Hefeeda and Majid Bagheri, “ Forest Fire Modeling and Early Detection using Wireless Sensor Networks," In. Adhoc \& Sensor Wireless Networks, Old City Publishing, pp.169-224 , 2009.

[15] N. Li, J. Hou, C.Sha, and L. Sha, "Design and analysis of an MST-based topology control algorithm," In. IEEE Trans. Wireless Commun., pp. 1195-1206, 2005.

[16] N. Christofides, "Worst-case analysis of a new heuristic for the travelling salesman problem," In. Technical Report 388, Graduate School of Industrial Administration, Carnegie Mellon University, 1976. 